\title{
Rheology of Cross-Linked Poly(Sodium Acrylate)/Sodium Silicate Hydrogels
}

\author{
Joanna Mastalska-Popławska ${ }^{1}$ - Piotr Izak ${ }^{1}$ • Lukasz Wójcik ${ }^{1}$ • \\ Agata Stempkowska ${ }^{2}$
}

Received: 8 July 2015 / Accepted: 20 October 2015 / Published online: 5 November 2015

(C) The Author(s) 2015. This article is published with open access at Springerlink.com

\begin{abstract}
Transparent hydrogels consisting of poly(sodium acrylate) and sodium silicate were synthesized by freeradical polymerization of sodium acrylate (ANa) in an aqueous solution of sodium silicate (i.e. water glass) with the silicate modulus (M) 2.50 and the Midafen R-102 polymer filler, in the presence of sodium thiosulphate/potassium persulphate (NTS/KPS) as the redox initiators and $N, N^{\prime}$ methylenebisacrylamide as the cross-linking monomer. The hydrogels, obtained in this way, were rheologically tested, and also the gelling point, dependence of shear stress on shear rate, and oscillation constants were determined. The results indicated that during the cross-linking reaction, which is associated with a gradual building of the three-dimensional gel structure, the reaction mixture changes its rheological behaviour from pseudo-thixotropic at the beginning of the reaction to pseudo- anti-thixotropic in the end. Both elasticity modulus $G^{\prime}$ and viscosity modulus $G^{\prime \prime}$ during the reaction and for the cast samples after $24 \mathrm{~h}$ have practically the same values and the phase shift angle $\delta$ is below $20^{\circ}$ which means that we obtained a highly elastic material. It was also found that the elasticity modulus $G^{\prime}$ values increase with the content of sodium silicate in the sample.
\end{abstract}

Joanna Mastalska-Popławska

jmast@agh.edu.pl

1 Faculty of Materials Science and Ceramics, AGH University of Science and Technology, Kraków, Poland

2 Faculty of Mining and Geoengineering, AGH University of Science and Technology, Kraków, Poland
Keywords Poly(sodium acrylate) - Water glass . Cross-linked hydrogels $\cdot$ Rheology of hydrogels · Oscillation rheology

\section{Introduction}

Polymer hydrogels can absorb and preserve a large amount of water, and this fact is used in many fields, such as tissue engineering, biosensors, drug delivery systems, or even horticulture and building construction. A three-dimensional structure of the gel is formed physically (van der Waals interactions, hydrogen bonds, electrostatic interaction) or chemically. The addition of different substances, for example laponite, mineral clays, soluble alkaline silicates, can significantly improve hydrogel properties, such as adhesion, mechanical strength, and absorption [1-8].

In this paper, we present the rheological characterization of acrylate hydrogels modified with the water solution of sodium silicate. The main emphasis was put on the rheological measurements during the cross-linking process and the description of the viscoelastic properties of the hydrogel samples during and after gelation.

To determine the gelling time (gel point), next to oscillation rheology, we also used the flow measurement (correlation of shear stress to changing shear rates), which is used to analyse the flow rheological properties of complete polymer solutions [9-11].

Polymer solutions are usually examples of pseudo-plastic fluids which means that their apparent viscosity decreases with the shear rate. The relationship between the shear stress versus shear rate is described by the empirical equation for a limited range so sometimes it is difficult to clearly explain the occurring phenomena. The two-parameter Ostwald de 
Waele power-law equation is the simplest and commonly used model:

$\tau=K \dot{\gamma}^{n}$

where $K$-consistency factor $(-), n$-flowing factor $(-)$, $\tau$ - shear stress (mPa.s), $\gamma$ - shear rate $\left(\mathrm{s}^{-1}\right)$, and $\eta$-apparent viscosity $(\mathrm{mPa})$.

But this equation does not meet the boundary conditions (for $n=1$ at $\gamma=0$ and $\eta=\infty$ ); therefore, other rheological models were developed with more parameters such as the three-parameter Ellis' formula and the four-parameter Meter's formula. Since they describe the behaviour of pseudo-plastic fluids where the yield point is caused by a reversible reaction associated with an intermolecular interaction, they are not recommended to describe the properties of pseudo-rheounstable systems [12,13].

If our goal is to determine the gel point (GP), the moment at which the phase transition occurs to a viscoelastic solid, the best are the oscillation measurements (dynamic rheology) in the range of non-destructive low frequencies [2,14-17]. The changes of $G^{\prime}$ elasticity (energy storage) modulus and $G^{\prime \prime}$ viscosity (energy loss) modulus are determined by the dependence of a small amplitude oscillatory shear as a function of cross-linking time at specified angular frequency $(\omega)$. At the beginning of the reaction, the $G^{\prime \prime}$ modulus is larger than $G^{\prime}$, at the gel point $G^{\prime}=G^{\prime \prime}$ and $G^{\prime}$ is much larger than $G^{\prime \prime}$ after the completion of the gelation [9-11,18].

\section{Experimental Section}

\subsection{Materials}

Sodium water glass (WG) with the silicate modulus $M=$ 2.50 (known under the trade name as sodium water glass R-145) was obtained from the Rudniki Chemical Plant (Rudniki, Poland). Midafen R-102 (MR-102), used in this experiment as a polymer filler, came from Lubrina SA, Poland.

The $20 \mathrm{wt} \%$ aqueous solution of sodium acrylate (ANa) was used as the monomer solution and laboratory synthesized (acrylic acid was manufactured by Lach-Ner, (Ner- atovice, Czech Republic) and sodium hydroxide came from Stanlab SJ (Poland)). The cross-linking agent $N, N^{\prime}$ methylenebisacrylamide (NNMBA) and the initiators of the polymerization reaction, potassium persulphate (KPS), and sodium thiosulphate (NTS) were purchased from Avantor Performance Materials Poland SA. All reagents were used without further purification.

\subsection{Synthesis}

\subsubsection{Synthesis of Sodium Acrylate}

The sodium acrylate solution was prepared by neutralizing acrylic acid to $\mathrm{pH}=7$ with a stoichiometric amount of the $22 \mathrm{wt} \%$ aqueous solution of sodium hydroxide. Due to the exothermic character of the reaction, the mixture was cooled with ice and water in such a way that the reaction temperature did not exceed $40^{\circ} \mathrm{C}$ (otherwise sodium acrylate would precipitate from the solution, which underwent dissolution by adding a small volume of water). Before polymerization, the solution was diluted to $20 \%$.

The resultant solution of sodium acrylate is labelled as $20 \%$ ANa (Table 1).

\subsubsection{Synthesis of Hydrogels}

The first stage involved the preparation of base mixtures by spreading the specified amount of Midafen R-102 (MR-102) (5, 10 and $20 \mathrm{wt} \%$ ) in water glass (WG). The suspension prepared in such a way was vigorously stirred on a magnetic stirrer for $10 \mathrm{~min}$. The resulting mixtures constituted the silicate basis for polymerization of poly(sodium acrylate)/sodium silicate hydrogels. In the next step, $20 \mathrm{wt} \%$ aqueous solution of sodium acrylate $(\mathrm{ANa})$ was added to the system at mass ratio of $1: 1$ and $1: 2$ to the base mixture and again was vigorously stirred on a magnetic stirrer for $5 \mathrm{~min}$ to obtain a transparent and uniform mixture. Because of the high $\mathrm{pH}$ of about 11 of the base mixture caused by the presence of water glass, the higher amount of sodium acrylate caused precipitation of acrylates and as a result the failure of the cross-linking reaction. Then, the cross-linking monomer
Table 1 Compositions of the poly(sodium acrylate)/sodium silicate hydrogels

\begin{tabular}{lllllll}
\hline Sample symbol* $(\%)$ & WG $(\mathrm{g})$ & MR-102 $(\mathrm{g})$ & $20 \%$ ANa $(\mathrm{g})$ & KPS $(\mathrm{g})$ & NTS $(\mathrm{g})$ & NNMBA $(\mathrm{g})$ \\
\hline $1: 1 / 5$ & 14.25 & 0.75 & 15.00 & 0.05 & 0.05 & 0.10 \\
$1: 1 / 10$ & 13.5 & 1.50 & & & & \\
$1: 1 / 20$ & 12.00 & 3.00 & & & \\
$1: 2 / 5$ & 19.00 & 1.00 & 10.00 & & \\
$1: 2 / 10$ & 18.00 & 2.00 & & & \\
$1: 2 / 20$ & 16.00 & 4.00 & & & & \\
\hline
\end{tabular}

* Sample symbol: for e.g. 1:1/5\%-1:1 means a mass ratio of $20 \mathrm{wt} \%$ aqueous solution of sodium acrylate to a base mixture, which consists of sodium water glass R-145 and a polymer filler Midafen R-102 (5-20\%) 
(NNMBA) and redox initiators (KPS/NTS) were introduced in an amount of $0.3 \mathrm{wt} \%$ to the sample weight. Polymerization proceeded according to a radical mechanism. In order to carry out the two rheological tests, i.e. flow curve measurement and frequency sweep standard test, two identical sets of polymerization mixtures were prepared.

Concentrations of the monomer, cross-linking agent (NNMBA) and initiators (KPS/NTS), as well as the mixing speed, were chosen to obtain the best conditions for the polymerization process and homogeneity of the resulting samples.

The exact composition of the hydrogels is shown in Table 1.

\subsection{Rheological Measurements}

Both types of rheological measurements, i.e. flow curve tests over time and oscillation measurements, were performed with a Physica MCR-301 (Anton Paar) rheometer. A parallel plate PP50 made of stainless steel with a $50 \mathrm{~mm}$ diameter was used. During the flow measurements and during the frequency sweep standard tests during the cross-linking reaction, the gap between plates was set at a distance of $0.125 \mathrm{~mm}$, while during the oscillation measurements of the cast samples (we prepared round samples $1 \mathrm{~mm}$ thick and $60 \mathrm{~mm}$ wide), the gap was set at a distance of $1 \mathrm{~mm}$.

The flow measurements were taken for increasing and decreasing shear rates in the range of $2-50 \mathrm{~s}^{-1}$, in $10-\mathrm{min}$ intervals. Five tests were performed for each sample, starting the measurement immediately after addition of the crosslinking monomer NNMBA and initiators (KPS/NTS) to the polymerization mixture and ending the test after $40 \mathrm{~min}$.

The frequency sweep standard test was carried out on the second set of polymerization mixtures immediately after addition of the cross-linking monomer NNMBA and initiators (KPS/NTS). The same measurement was repeated $24 \mathrm{~h}$ later, on the cast hydrogel samples. Those systems were subjected to low shear stress, in the range of angular shear rates $500-0.05 \mathrm{~s}^{-1}$.

\subsection{Results and Discussion}

\subsection{Dependence of Shear Stress to Shear Rate (Flow Curves)}

Figures 1 and 2 show the dependence of shear stress on shear rate for two systems which are summarized in Table 1, 1:1/5 and 1:2/5\%, respectively. The filled points denote the curves plotted for the increasing shear rates, while the empty ones correspond to the decreasing shear rates. As we can see, the shape of the flow curve changes over time. At the beginning of the reaction ( $0 \mathrm{~min})$, in both cases we have a polymer solution which starts to polymerize within $10 \mathrm{~min}$. The hysteresis area increases with time and changes its value from positive to negative, and already after $10 \mathrm{~min}$ the system changes prob-
Fig. 1 Dependence of shear stress on shear rate over time for $1: 1 / 5 \%$ polymerization mixture

Fig. 2 Dependence of shear stress on shear rate over time for $1: 2 / 5 \%$ polymerization mixture
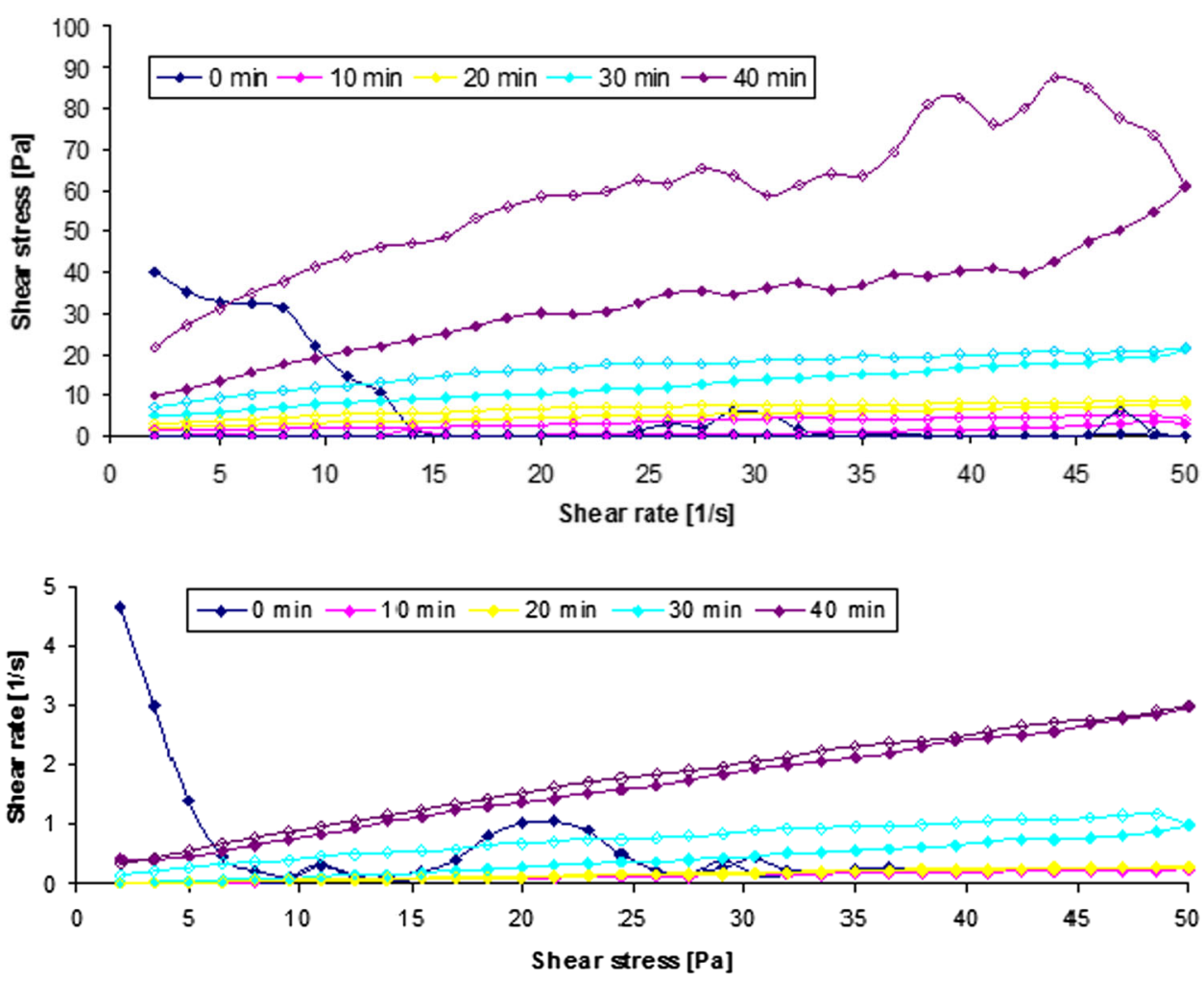
Table 2 Values of the hysteresis area $(\mathrm{Pa} / \mathrm{s})$ of the silicate- polymers mixtures during polymerization

\begin{tabular}{lrrrrr}
\hline Sample symbol $(\%)$ & \multicolumn{1}{c}{0 min } & $10 \min$ & $20 \min$ & $30 \min$ & \multicolumn{1}{c}{$40 \min$} \\
\hline $1: 1 / 5$ & 329.12 & -115.78 & -86.23 & -199.00 & -1426.56 \\
$1: 1 / 10$ & 63.51 & -5.77 & -18.74 & -52.51 & -168.90 \\
$1: 1 / 20$ & 617.49 & -9.54 & -36.34 & -55.15 & -739.53 \\
$1: 2 / 5$ & 29.53 & 0.41 & -0.82 & -17.03 & -5.82 \\
$1: 2 / 10$ & 12.79 & 0.20 & -4.23 & -3.61 & -7.56 \\
$1: 2 / 20$ & 3.31 & -0.68 & -6.28 & -66.51 & -114.90 \\
\hline
\end{tabular}

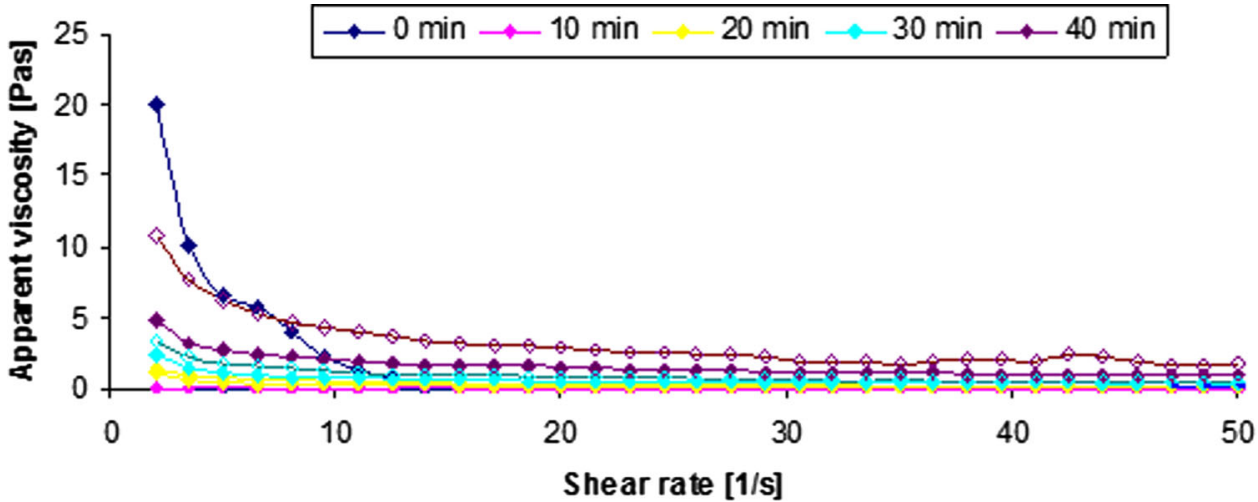

Shear rate $[1 / \mathrm{s}]$
Fig. 3 Dependence of apparent viscosity on shear rate over time for $1: 1 / 5 \%$ polymerization mixture ably to pseudo-anti-thixotropic which may indicate that the cross-linking reaction is starting.

The courses of flow curves of other polymerization mixtures are similar.

To confirm these observations, we have placed the values of the hysteresis area in Table 2. They were determined by the use of the integral method, i.e. definite integrals in the range $2-50 \mathrm{~s}^{-1}$ were calculated on the basis of equations of the trend curves of decreasing and increasing flow curves. According to the literature [13], these values constitute the measure of thixotropy (structure destruction) or anti-thixotropy (structure reconstruction) and correspond to the internal energy of the system used for the destruction or reconstruction of the suspension structure during the measurement, i.e. during shearing at different rates. Comparing only these results we can say that the values of the hysteresis area are much bigger for the 1:1 polymerization system than for the 1:2 polymerization system which indicates that the higher the content of sodium acrylate solution, the more cross-linked hydrogel we get.

As it was mentioned earlier in this chapter, the considered polymer- silicate hydrogels are pseudo-plastic fluids, of which shear thinning is the characteristic feature. This effect occurs at both decreasing and increasing shear rates (Fig. 3). It is probably associated with breaking of the intermolecular bonds during the measurement. In this respect, efforts were made to fit an appropriate mathematical model describing the rheological behaviour of the tested mixtures.
Due to the complexity of the system, i.e. its variable pseudo-plastic character occurring during polymerization, we had to apply the generalized model of the flow curve. We did it by averaging the measurement points of the flow curves obtained during the flow tests. This allowed us to specify some important usable features of our hydrogels. The tested hydrogels have a yield point increasing with time, which indicates the progression of the cross-linking reaction. For the $1: 1 / 5 \%$ sample, which is shown in Fig. 4, the values are in the range of $0.70-15.5 \mathrm{~Pa}$. The equivalent composition of $1: 2$ has the yield point in the range of $0.02-0.38 \mathrm{~Pa}$.

On the basis of rheological equations of state fitted to the conventional models of rheostable fluids, the best results were obtained for the two-parameter Ostwald de Waele power model. As we know, this is the simplest model used to characterize the behaviour of polymer solutions which describes shear-thinning fluids (Eq. 1.1). The fitted parameters are presented in Table 3.

A specific dependence between correlation coefficient $R^{2}$ (correlation coefficient) and time of polymerization was observed. At the beginning of the reaction $(0 \mathrm{~min}), R^{2}$ is at zero and gradually rises over time. After $20 \mathrm{~min}$, its value oscillates around $90 \%$ for $1: 1$ ratio, whereas for 1:2 ratio, this level is achieved faster within $10 \mathrm{~min}$. Below these times, the processes associated with shearing of the polymer mixture predominate, whereas the chemical reactions associated with polymerization and crosslinking of the hydrogel dominate afterwards. The val- 


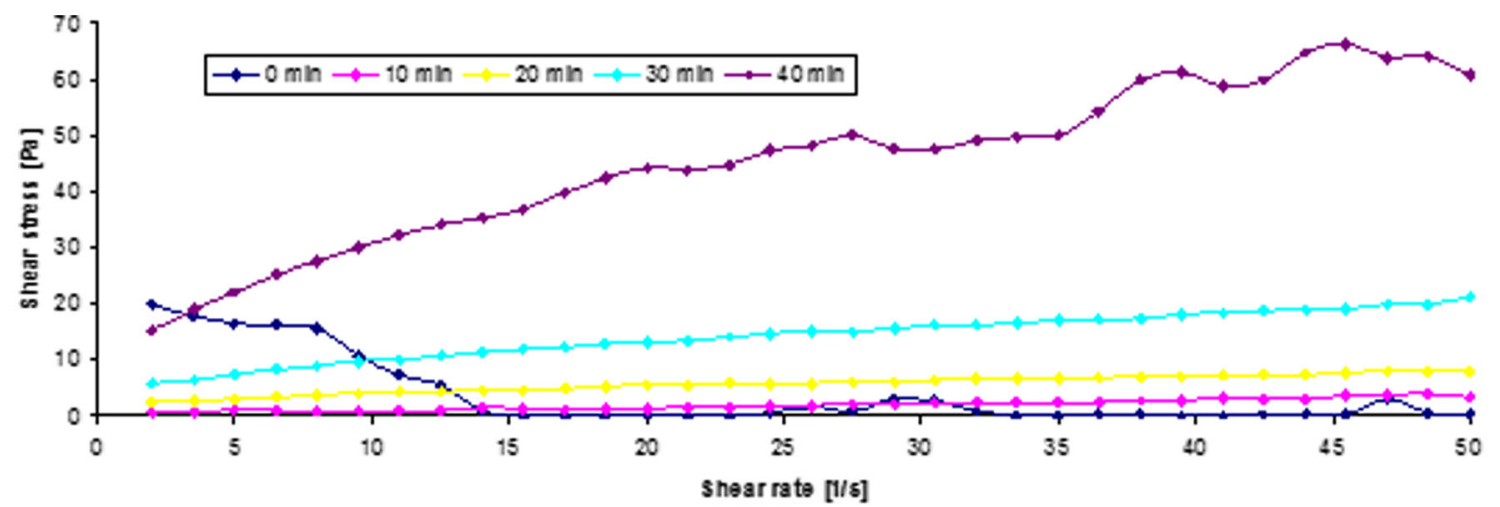

Fig. 4 Generalized model of the flow curve for 1:1/5\% polymerization mixture

Table 3 Material constants of Ostwald de Waele power model

\begin{tabular}{|c|c|c|c|c|c|c|}
\hline \multirow{2}{*}{ Sample symbol (\%) } & \multirow{2}{*}{ Parameter } & \multicolumn{5}{|c|}{ Reaction time (min) } \\
\hline & & 0 & 10 & 20 & 30 & 40 \\
\hline \multirow[t]{3}{*}{$1: 1 / 5$} & $R^{2}$ & 0.000 & 0.302 & 0.790 & 0.816 & 0.371 \\
\hline & $K$ & 1.665 & 0.051 & 1.553 & 3.692 & 10.409 \\
\hline & $n$ & -0.552 & 1.035 & 0.414 & 0.428 & 0.444 \\
\hline \multirow[t]{3}{*}{$1: 1 / 10$} & $R^{2}$ & 0.000 & 0.633 & 0.944 & 0.906 & 0.849 \\
\hline & $K$ & 0.440 & 0.013 & 0.093 & 0.335 & 1.004 \\
\hline & $n$ & -0.249 & 0.819 & 0.855 & 0.745 & 0.693 \\
\hline \multirow[t]{3}{*}{$1: 1 / 20$} & $R^{2}$ & 0.000 & 0.176 & 0.866 & 0.832 & 0.722 \\
\hline & $K$ & 3.585 & 0.381 & 0.337 & 1.739 & 0.244 \\
\hline & $n$ & -0.647 & 0.577 & 0.733 & 0.389 & 0.695 \\
\hline \multirow[t]{3}{*}{$1: 2 / 5$} & $R^{2}$ & 0.000 & 0.302 & 0.790 & 0.816 & 0.371 \\
\hline & $K$ & 0.163 & 0.051 & 1.553 & 3.692 & 10.409 \\
\hline & $n$ & 0.117 & 1.035 & 0.414 & 0.428 & 0.444 \\
\hline \multirow[t]{3}{*}{$1: 2 / 10$} & $R^{2}$ & 0.000 & 0.981 & 0.948 & 0.977 & 0.972 \\
\hline & $K$ & 0.122 & 0.012 & 0.016 & 0.057 & 0.127 \\
\hline & $n$ & 0.238 & 0.874 & 0.922 & 0.771 & 0.701 \\
\hline \multirow[t]{3}{*}{$1: 2 / 20$} & $R^{2}$ & 0.017 & 0.935 & 0.948 & 0.855 & 0.911 \\
\hline & $K$ & 0.111 & 0.038 & 0.041 & 0.118 & 0.259 \\
\hline & $n$ & 0.329 & 0.672 & 0.814 & 1.037 & 1.052 \\
\hline
\end{tabular}

ues of flow coefficient $n$ are below 1 or a little over it and determine the pseudo-plastic nature of the respondent hydrogels.

\subsection{Oscillation Measurements}

Dynamic rheology measurements provide information about the cross-linking kinetics by determining the gel point (crossover point) during the reaction and the influence of the composite composition on the viscoelastic properties of the poly(sodium acrylate)/sodium silicate hydrogels.

Figures 5 and 6 show the dependence of $G^{\prime}$ and $G^{\prime \prime}$ modulus of angular frequency during the cross-linking reaction. For both mass ratios (Table 1), the storage modulus $G^{\prime}$ is much larger than the loss modulus $G^{\prime \prime}$ in the plateau region and the gel point (GP) is achieved very fast. For the 1:1 mass ratio, the gel point occurs between 250 and $232 \mathrm{~s}^{-1}$, whereas for the 1:2 mass ratio, where we have a larger content of sodium silicate, the range where the gel point occurs is slightly wider, $163-136 \mathrm{~s}^{-1}$.

The second frequency sweep standard test was carried out to check the influence of the mass ratio of the poly(sodium acrylate) to the base mixture on the viscoelastic properties of the tested samples after the cross-linking reaction. As it is shown in Figs. 7 and 8, the storage (elasticity) modulus $G^{\prime}$ and the loss (viscous) modulus $G^{\prime \prime}$ strongly depend on the sample composition and $G^{\prime}$ is higher than $G^{\prime \prime}$ which means that we have more elastic than viscous hydrogels. The curves of $G^{\prime}$ and $G^{\prime \prime}$ crossed each other and the crossover point $\left(G^{\prime}=G^{\prime \prime}\right)$ shifted to lower frequencies with the decreasing 
Fig. 5 Shear modulus as a function of angular frequency during cross-linking reaction for 1:1 mass ratio

Fig. 6 Shear modulus as a function of angular frequency during cross-linking reaction for 1:2 mass ratio

Fig. 7 Dependence of the $G^{\prime}$ and $G^{\prime \prime}$ modules on the angular frequency for the 1:1 mass ratio
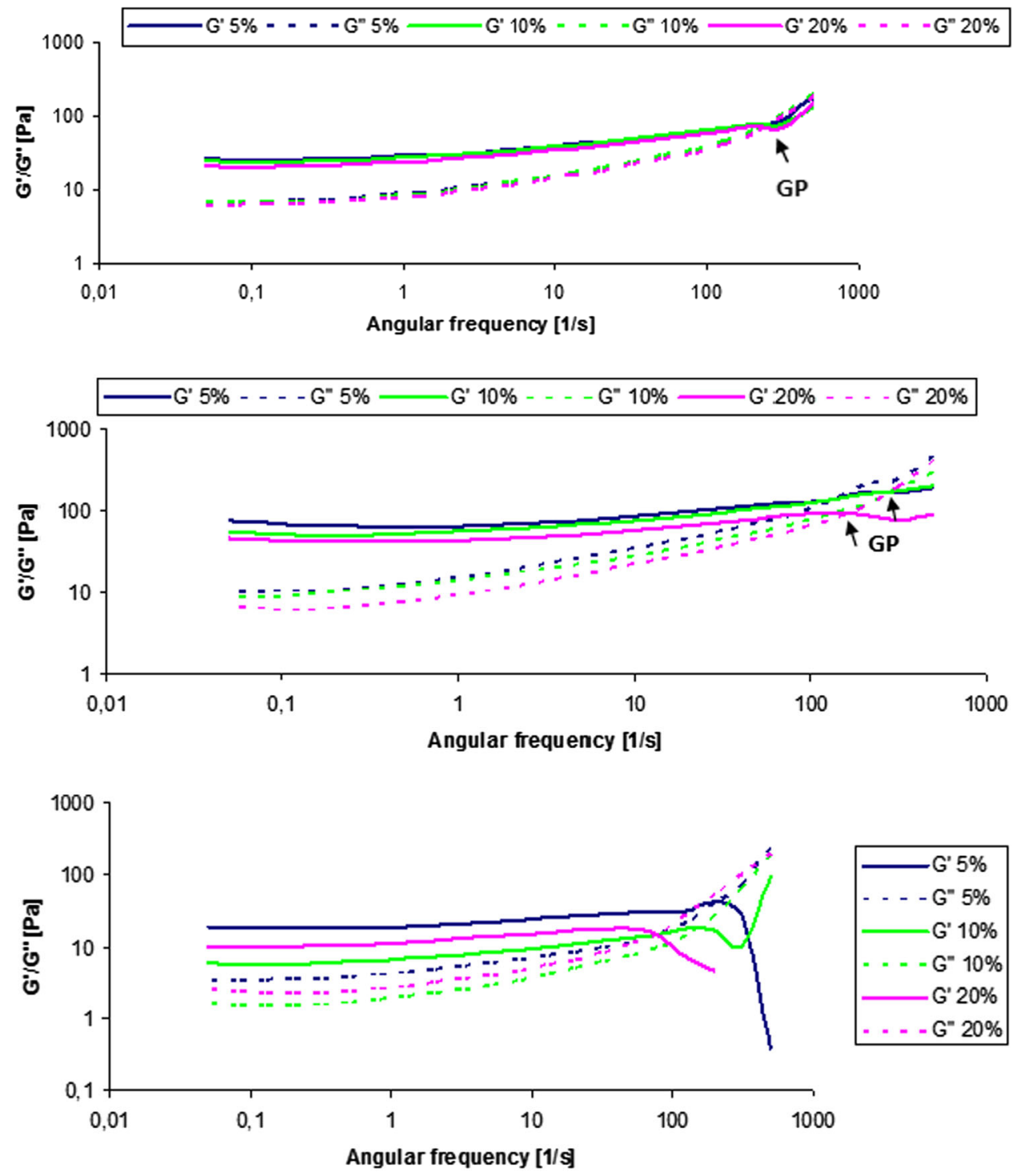

Fig. 8 Dependence of the $G^{\prime}$ and $G^{\prime \prime}$ modules on the angular frequency for the 1:2 mass ratio

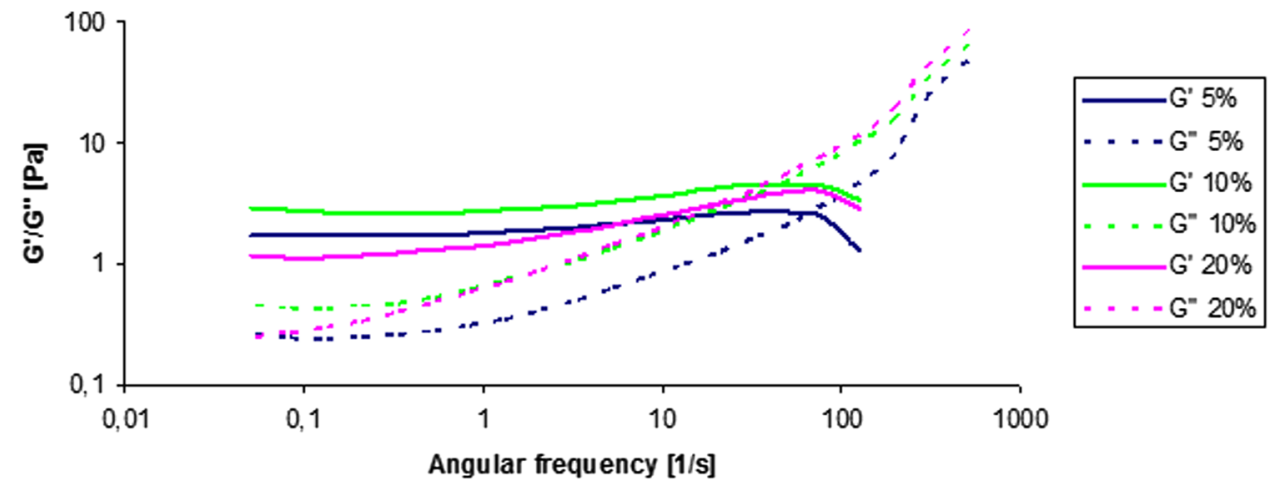

content of poly(sodium acrylate) or, in other words, with the increasing content of water glass.

For both mass ratios, the value of $G^{\prime}$ and $G^{\prime \prime}$ decreases with the increasing content of Midafen R-102 in a base mixture. Comparing the values of $G^{\prime}$ and $G^{\prime \prime}$ with the poly- mer content, it was found that less poly(sodium acrylate) was present, the value of both moduli was higher. Only at high frequencies (higher than $100 \mathrm{rad} / \mathrm{s}$ ), $G^{\prime \prime}$ is larger than $G^{\prime}$ which means that in this range, the viscous properties predominate. 
Table 4 Values of oscillation constants during the cross-linking reaction $(A)$ and for cast samples $(B)$

\begin{tabular}{|c|c|c|c|c|c|c|c|c|c|}
\hline \multirow[t]{2}{*}{ MR-102 (wt\%) } & \multirow[t]{2}{*}{ Mass ratio } & \multicolumn{2}{|c|}{$G^{\prime}(\mathrm{Pa})$} & \multicolumn{2}{|c|}{$G^{\prime \prime}(\mathrm{Pa})$} & \multicolumn{2}{|l|}{$\underline{\operatorname{tg}(\delta)}$} & \multicolumn{2}{|l|}{$\delta\left(^{\circ}\right)$} \\
\hline & & $A$ & $B$ & $A$ & $B$ & $A$ & $B$ & $A$ & $B$ \\
\hline \multirow[t]{2}{*}{5} & $1: 1$ & 26.37 & 26.13 & 7.27 & 7.27 & 0.27 & 0.28 & 15.11 & 15.64 \\
\hline & $1: 2$ & 67.91 & 63.85 & 10.37 & 10.87 & 0.15 & 0.17 & 8.53 & 9.65 \\
\hline \multirow[t]{2}{*}{10} & $1: 1$ & 24.44 & 24.60 & 6.97 & 7.23 & 0.28 & 0.29 & 15.64 & 16.20 \\
\hline & $1: 2$ & 54.04 & 51.30 & 9.61 & 9.28 & 0.18 & 0.18 & 10.20 & 10.20 \\
\hline \multirow[t]{2}{*}{20} & $1: 1$ & 20.98 & 20.85 & 6.54 & 6.54 & 0.31 & 0.31 & 17.22 & 17.22 \\
\hline & $1: 2$ & 43.04 & 44.17 & 7.05 & 6.77 & 0.16 & 0.15 & 9.09 & 8.53 \\
\hline
\end{tabular}

The above assumption is confirmed by the oscillation constant of $G^{\prime}$ and $G^{\prime \prime}$ moduli and phase angle $\delta$ (Table 4). It was noticed that $G^{\prime}$ and $G^{\prime \prime}$ values do not depend on time and are higher for the 1:2 mass ratio. Phase angle $\delta$ is low, below $20^{\circ}$, which indicates that we obtained highly elastic hydrogels $\left(90^{\circ}\right.$ denotes a totally viscous substance and $0^{\circ}$ is related to completely elastic systems $[5,14,19])$.

\section{Conclusions}

In this study, the rheological behaviour of the hydrogel during the polymerization reaction and viscoelastic properties of the cast poly(sodium acrylate)/sodium silicate hydrogel samples were studied. We have compared two methods for determining the gelation point, i.e. the oscillation frequency sweep standard test and the flow curve measurement, normally used to determine the flow behaviour of rheostable fluids.

We found a correlation between the changing thixotropic properties of the formed gel to its transition from the sol to the gel. At the beginning of the cross-linking reaction, for both mass ratios 1:1 and 1:2 of the sodium acrylate monomer solution to the base mixture of Midafen R-102 polymer filler and sodium water glass, the flow curves exhibited pseudothixotropic behaviour with a small hysteresis area, which is characteristic for a diluted polymer solution, and in 10min time the observed properties changed to pseudo-antithixotropic with a larger hysteresis area and a negative value. This can be explained by the fact of the proceeding crosslinking reaction. Pseudo-anti-thixotropic behaviour means that the chemical reaction causes irreversible changes and the cross-linking reaction is faster than the deconstruction of the occurring gel caused by shear stress.

To confirm the above considerations, we were trying to fit an appropriate rheological model. As it was mentioned before in this paper, mathematical models usually describe systems which undergo reversible changes caused by the intermolecular interactions. In the case of the rheounstable fluids, we had to use the generalized model of the flow curve. The best fit was obtained for the Ostwald de Waele power-law model. Analysing the value of the correlation coefficient $R^{2}$, the highest fit was found after $20 \mathrm{~min}$ for the 1:1 mass ratio and after $10 \mathrm{~min}$ for the 1:2 mass ratio. We defined these values as the gelling point.

The oscillation method measurements have shown that the obtained silicate-polymer hydrogels are highly elastic, which is confirmed by the value of the phase angle shift and the fact that the values of the elasticity modulus $G^{\prime}$ are larger than the values of the viscosity modulus $G^{\prime \prime}$. The values of both moduli decrease with the amount of Midafen R-102 in the base mixture, and they were significantly higher for the hydrogel samples of mass ratio 1:2 of the poly(sodium acrylate) to a base mixture.

Summing up, the flow curve measurements give the similar information about kinetics of the cross-linking reaction as the oscillation frequency sweep standard test, typically used for this purpose. Also, the value of the power correlation coefficient $R^{2}$ for the chosen Ostwald de Waele rheological model can describe the cross-linking kinetics, particularly in relation to changes of consistency $(K)$ and shear-thinning phenomena $(n)$.

Open Access This article is distributed under the terms of the Creative Commons Attribution 4.0 International License (http://creativecommons. org/licenses/by/4.0/), which permits unrestricted use, distribution, and reproduction in any medium, provided you give appropriate credit to the original author(s) and the source, provide a link to the Creative Commons license, and indicate if changes were made.

\section{References}

1. Shen, M.; Li, L.; Sun, Y. et al.: Rheology and adhesion of poly(acrylic acid)/laponite nanocomposite hydrogels as biocompatible adhesives. Langmuir 30, 1636-1642 (2014)

2. Winter, H.H.: Evolution of rheology during chemical gelation. Prog. Colloid Polym. Sci. 75, 104-110 (1987)

3. Valle, F.; Muller, C.; Durand, A. et al.: Synthesis and rheological properties of hydrogels based on amphiphilic alginate-amide derivatives. Carbohydr. Res. 344, 223-228 (2009)

4. Grattoni, C.A.; Al-Sharji, H.H.; Yang, C. et al.: Rheology and permeability of crosslinked polyacrylamide gel. J. Colloid Interface Sci. 240, 601-607 (2001)

5. Cicha-Szot, R.; Falkowicz, S.: Influence of modifier on the viscoelastic properties of silicate gels. Oil-Gas 12, 1102-1108 (2010) (in Polish) 
6. Yang, J.; Shi, F.K.; Gong, C. et al.: Dual cross-linked networks hydrogels with unique swelling behavior and high mechanical strength: based on silica nanoparticle and hydrophobic association. J. Colloid Interface Sci. 381, 107-115 (2012)

7. Jin, Q.; Schexnaidler, P.; Gaharwar, A.K.; Schmidt, G.: Silicate cross-linked bio-nanocomposite hydrogels from PEO and Chitosan. Macromol. Biosci. 9, 1028-1035 (2009)

8. Gaharwar, A.K.; Rivera, C.P.; Wu, C.J.; Schmidt, G.: Transparent, elastomeric and tough hydrogels from poly(ethylene glycol) and silicate nanoparticles. Acta Biomater. 7, 4139-4148 (2011)

9. Malana, M.A.; Zohra, R.; Khan, M.S.: Rheological characterization of novel physically crosslinked terpolymeric hydrogels at different temperatures. Korea-Aust. Rheol. J. 24(3), 155-162 (2012)

10. Kelessidis, V.C.; Maglione, R.; Tsamantaki, C.; Aspirtakis, Y.: Optimal determination of rheological parameters for HerschelBulkley drilling fluids and impact on pressure drop, velocity profiles and penetration rates during drilling. J. Pet. Sci. Eng. 53, 203224 (2006)

11. Hammadi, L.; Ponton, A.; Belhadri, A.: Temperature effect on shear flow and thixotropic behavior of residual sludge from wastewater treatment plant. Mech. Time-Depend. Mater. 17, 401-412 (2013)

12. Al-Zahrani, S.M.: A generalized rheological model for shear thinning fluids. J. Pet. Sci. Eng. 17, 211-215 (1997)
13. Izak, P.: Rheology of Ceramic Slurries. AGH Publishing House, Krakow (2012) (in Polish)

14. Prud'homme, R.K.; Uhl, J.T.; Poinsatte, J.P.; Halverson, F.: Rheological monitoring of the formation of polyacrylamide/ $\mathrm{Cr}^{3+}$ gels. Soc. Pet. Eng. J. 10, 804-808 (1983)

15. De Rosa, M.E.; Winter, H.H.: The effect of entanglements on the rheological behavior of polybutadiene critical gels. Rheol. Acta 33, 220-237 (1994)

16. Harini, M.; Desphande, A.P.: Rheology of poly (sodium acrylate) hydrogels during cross-linking with and without cellulose microfibrils. J. Rheol. 53(1), 31-47 (2009)

17. Winter, H.H.: Can the gel point of a cross-linking polymer be detected by the $G^{\prime}-G^{\prime \prime}$ Crossover. Polym. Eng. Sci. 27(22), 16981702 (1987)

18. Mezger, T.: The rheology handbook: for users of rotational and oscillatory rheometers. Vincentz Network GmbH \& Co KG, Hannover (2002)

19. Nesrinne, S.; Djamel, A.: Synthesis, characterization and rheological behavior of $\mathrm{pH}$ sensitive poly(acrylamide-co-acrylic acid) hydrogels. Arab. J. Chem. 3-6 (2013). doi:10.1016/j.arabjc.2013. 11.027 